\title{
Efficient Simulation for Pricing Barrier Options with Two-Factor Stochastic Volatility and Stochastic Interest Rate
}

\author{
Zhang Sumei and Zhao Jieqiong \\ School of Science, Xi'an University of Posts and Telecommunications, Xi'an 710121, China \\ Correspondence should be addressed to Zhang Sumei; zhanggsumei@sina.com
}

Received 11 August 2017; Accepted 8 October 2017; Published 14 November 2017

Academic Editor: Fazal M. Mahomed

Copyright (c) 2017 Zhang Sumei and Zhao Jieqiong. This is an open access article distributed under the Creative Commons Attribution License, which permits unrestricted use, distribution, and reproduction in any medium, provided the original work is properly cited.

\begin{abstract}
This paper presents an extension of the double Heston stochastic volatility model by combining Hull-White stochastic interest rates. By the change of numeraire and quadratic exponential scheme, this paper develops a new simulation scheme for the extended model. By combining control variates and antithetic variates, this paper provides an efficient Monte Carlo simulation algorithm for pricing barrier options. Based on the differential evolution algorithm the extended model is calibrated to S\&P 500 index options to obtain the model parameter values. Numerical results show that the proposed simulation scheme outperforms the Euler scheme, the proposed simulation algorithm is efficient for pricing barrier options, and the extended model is flexible to fit the implied volatility surface.
\end{abstract}

\section{Introduction}

A barrier option is a path-dependent option which is exterminated (knocked out) or initiated (knocked in) if the underlying spot price hits the specified barrier level during the life of the option. Because of this supplementary risk, barrier options are cheaper than plain vanilla options and thus are widely traded in exchanges worldwide. One-factor stochastic volatility models can generate "smile," leverage effects, and term structure effects which cannot be explained by the Black-Scholes model $[1,2]$. Consequently many papers [3-9] evaluate barrier options under one-factor stochastic volatility models.

However, one-factor stochastic volatility models including the Heston model [10] present the poor performance when fitting the stiff volatility skews [11]. One extension by using multiple stochastic volatility factors has been presented in some literatures [2, 12, 13]. Christoffersen et al. [2] confirm that the double Heston model significantly improves the flexibility of the one-factor stochastic volatility model in capturing the volatility term structure. In addition, stochastic interest rate is crucial for option pricing because it ensures proper discounting of future payoffs. In recent literatures [1417], Hull-White stochastic interest rate which is analytically tractable has been incorporated into one-factor stochastic volatility model for pricing path-dependent options. Therefore, the model which incorporates multifactor stochastic volatility and stochastic interest rate may be more reasonable for pricing barrier options.

Barrier options with less stochastic factors can be efficiently evaluated by partial differential equation (PDE) methods $[3,4,18,19]$. However, the evaluation of barrier options with multiple stochastic factors is to solve a highdimensional PDE which makes PDE methods quite complex and potentially prone to accuracy and stability problems [1]. A more efficient method for pricing barrier options in this case is the Monte Carlo method; see [20, 21]. The main purpose of this paper is to provide a Monte Carlo method for pricing barrier options under a two-factor stochastic volatility and stochastic interest rate model.

The main contributions of this paper are threefold. Firstly, this paper extends the double Heston model to stochastic interest rate. Secondly, this paper provides a new simulation scheme for the extended model. Thirdly, the paper develops an efficient Monte Carlo algorithm for pricing barrier options. The rest of the paper is organized as follows. Section 2 presents the extended model. Section 3 details the simulation scheme for the proposed model. Section 4 develops the 
simulation algorithm for pricing barrier options. Section 5 provides numerical experiments. Section 6 concludes.

\section{The Pricing Model}

Let $\left\{\Omega, \mathscr{F},\left\{\mathscr{F}_{t}\right\}, P\right\}$ be a complete probability space, where $P$ is a risk-neutral bank account measure. Assume that $W_{j}^{S}(t)$, $W_{j}^{V}(t)(j=1,2)$, and $W^{r}(t)$ are all standard Brownian motions which are $\mathscr{F}_{t}$-adapted. The double Heston model proposed by Christoffersen et al. [2] is defined by the following stochastic partial differential equations:

$$
\begin{aligned}
& d S(t)=r S(t) d t+\sum_{j=1}^{2} \sqrt{V_{j}(t)} S(t) d W_{j}^{S}(t), \\
& d V_{1}(t)=b_{1}\left(\theta_{1}-V_{1}(t)\right) d t+\sigma_{1} \sqrt{V_{1}(t)} d W_{1}^{V}(t), \\
& d V_{2}(t)=b_{2}\left(\theta_{2}-V_{2}(t)\right) d t+\sigma_{2} \sqrt{V_{2}(t)} d W_{2}^{V}(t),
\end{aligned}
$$

where $r$ is constant interest rate, $W_{j}^{S}(t)$ and $W_{j}^{V}(t)(j=1,2)$ are correlated with parameter $\rho_{j} . b_{j}, \theta_{j}, \sigma_{j}$ are the mean reversion speed, long-term volatility level, and volatility of process $V_{j}(t)(j=1,2)$, respectively. $2 b_{j} \theta_{j} \geq \sigma_{j}^{2}$ are supposed to make the processes $V_{j}(t)$ remain strictly positive. Conditional on $V_{j}(t), V_{j}(t+\Delta)$ obey $\sigma_{j}^{2}\left(1-e^{-b_{j} \Delta}\right) / 4 b_{j}$ times a noncentral $\chi^{2}$ distribution with $d_{j}$ degree of freedom and noncentrality parameter $\xi_{j}$; that is,

$$
\begin{aligned}
& P\left(V_{j}(t+\Delta) \leq x_{j} \mid V_{j}(t)\right) \\
& \quad=F_{\chi_{d_{j}}^{2}\left(\xi_{j}\right)}\left(\frac{4 b_{j}}{\sigma_{j}^{2}\left(1-e^{-b_{j} \Delta}\right)} x_{j}\right),
\end{aligned}
$$

where $d_{j}=4 b_{j} \theta_{j} / \sigma_{j}^{2}, \quad \xi_{j}=4 b_{j} e^{-b_{j} \Delta} V_{j}(t) / \sigma_{j}^{2}(1-$ $\left.e^{-b_{j} \Delta}\right)$, and $F_{\chi_{d_{j}}^{2}\left(\xi_{j}\right)}\left(z_{j}\right)=\sum_{i=1}^{\infty}\left(\xi_{j}^{i} e^{-\xi_{j} / 2} / 2^{d_{j} / 2+2 i} i ! \Gamma\left(i+d_{j} /\right.\right.$ 2)) $\int_{0}^{z_{j}} z_{j}^{d_{j} / 2+i-1} e^{-v / 2} d v$.

We consider the Hull-White stochastic interest rate [22] which is driven by the following mean-reverting process:

$$
d r(t)=\left(\theta(t)-b_{r} r(t)\right) d t+\sigma_{r} d W^{r}(t),
$$

where positive constants $b_{r}, \sigma_{r}$ are the mean reversion speed and volatility of process $r(t)$, respectively. $\theta(t)>0$ is used to fit the initial term structure of process $r(t)$. Following Brigo and Mercurio [23], the price of a zero-coupon bond maturing at time $T$ can be formulated as follows:

$$
P(t, T)=A(t, T) \exp [-B(t, T) r(t)],
$$

where

$$
\begin{aligned}
& A(t, T)=\frac{P(0, T)}{P(0, t)} \\
& \cdot \exp \left[B(t, T) f(0, t)-\frac{\sigma_{r}^{2}}{4 b_{r}}\left(1-e^{-2 b_{r} t}\right) B(t, T)^{2}\right],
\end{aligned}
$$

$$
\begin{aligned}
& B(t, T)=\frac{1-e^{-b_{r}(T-t)}}{b_{r}}, \\
& f(0, t)=-\frac{\partial \ln P(0, t)}{\partial t} .
\end{aligned}
$$

We replace constant $r$ in (1) with Hull-White stochastic interest rate (3) and define the double Heston Hull-White (DHHW) model by a four-dimensional system of stochastic differential equations:

$$
\begin{aligned}
& d S(t)=r(t) S(t) d t+\sum_{j=1}^{2} \sqrt{V_{j}(t)} S(t) d W_{j}^{S}(t), \\
& d V_{1}(t)=b_{1}\left(\theta_{1}-V_{1}(t)\right) d t+\sigma_{1} \sqrt{V_{1}(t)} d W_{1}^{V}(t), \\
& d V_{2}(t)=b_{2}\left(\theta_{2}-V_{2}(t)\right) d t+\sigma_{2} \sqrt{V_{2}(t)} d W_{2}^{V}(t), \\
& d r(t)=\left(\theta(t)-b_{r} r(t)\right) d t+\sigma_{r} d W^{r}(t) .
\end{aligned}
$$

Suppose $V_{1}(0)=V_{1}, V_{2}(0)=V_{2}, r(0)=r$, and $S(0)=$ $S$. Assume that any two random processes are uncorrelated with each other except $\operatorname{cov}\left(d W_{1}^{S}(t), d W_{1}^{V}(t)\right)=\rho_{1} d t$, $\operatorname{cov}\left(d W_{2}^{S}(t), d W_{2}^{V}(t)\right)=\rho_{2} d t$.

\section{Simulation Scheme for the DHHW Model}

To reduce the dimension of the Monte Carlo simulation, we change from the measure $P$ to the $T$-forward measure $Q$ by using $P(0, T)$ as numeraire. Set

$$
z(t, T)=\ln \frac{S(t)}{P(t, T)} .
$$

By the Itô formula, we rewrite (6) as follows:

$$
\begin{aligned}
d z(t, T)= & -\frac{1}{2}\left[V_{1}(t)+V_{2}(t)+\sigma_{r}^{2} B^{2}(t, T)\right] \\
& +\sum_{j=1}^{2} \sqrt{V_{j}(t)} W_{j}^{S}(t)+\sigma_{r} B(t, T) W^{r}(t) .
\end{aligned}
$$

3.1. Variance Simulation. Based on the fact that a noncentral $\chi^{2}$ distribution with high noncentrality parameter can be well approximated by a normal distribution, we use quadratic exponential scheme [24] for discrete variance process $V_{j}(t)$.

By (2) and simple calculation, we have

$$
\begin{aligned}
m_{j} & =E\left[V_{j}(t+\Delta) \mid V_{j}(t)\right]=\theta_{j}+\left(V_{j}(t)-\theta_{j}\right) e^{-b_{j} \Delta}, \\
s_{j}^{2} & =V\left[V_{j}(t+\Delta) \mid V_{j}(t)\right] \\
& =\frac{\sigma_{j}^{2} e^{-b_{j} \Delta} V_{j}(t)}{b_{j}}\left(1-e^{-b_{j} \Delta}\right)+\frac{\theta_{j} \sigma_{j}^{2}}{2 b_{j}}\left(1-e^{-b_{j} \Delta}\right)^{2} .
\end{aligned}
$$

Set $\psi_{j}=s_{j}^{2} / m_{j}^{2}$. Provided that $\psi_{j} \leq 2$ and $\xi_{j} \rightarrow \infty$, we approximate $V_{j}(t+\Delta)$ (the segment of high value) by

$$
V_{j}(t+\Delta) \approx \alpha_{j}\left(\beta_{j}+Z_{V j}\right)^{2}
$$


where $\alpha_{j}=m_{j} /\left(1+b_{j}^{2}\right), \beta_{j}^{2}=2 \psi_{j}^{-1}-1+2 \sqrt{2 \psi_{j}^{-1}} \sqrt{2 \psi_{j}^{-1}-1}$, and $Z_{V j}$ are independent standard normal random variables.

Provided that $\psi_{j} \geq 1$ and $\xi_{j} \rightarrow 0$, we approximate $V_{j}(t+$ $\Delta$ ) (the segment of low value) by

$$
\begin{aligned}
& V_{j}(t+\Delta) \\
& \approx \begin{cases}\frac{m_{j}\left(\psi_{j}+1\right)}{2} \log \frac{2}{\left(1-U_{j}\right)\left(1+\psi_{j}\right)}, & \frac{\psi_{j}-1}{\psi_{j}+1}<U_{j} \leq 1 \\
0, & 0 \leq U_{j} \leq \frac{\psi_{j}-1}{\psi_{j}+1},\end{cases}
\end{aligned}
$$

where $U_{j}$ are independent uniform random numbers.

3.2. Asset Price Simulation. Under the $T$-forward measure $Q$, by integrating (11) and applying the Cholesky decomposition, we rewrite asset price process (6) as follows:

$$
\begin{aligned}
z(t+ & \Delta, T) \\
= & z(t, T) \\
& -\frac{1}{2} \int_{t}^{t+\Delta}\left[V_{1}(u)+V_{2}(u)+\sigma_{r}^{2} B^{2}(u, T)\right] d u \\
& +\sum_{j=1}^{2} \rho_{j} \int_{t}^{t+\Delta} \sqrt{V_{j}(u)} d W_{j}^{V}(u) \\
& +\sum_{j=1}^{2} \sqrt{1-\rho_{j}^{2}} \int_{t}^{t+\Delta} \sqrt{V_{j}(u)} d W_{j}(u) \\
& +\sigma_{r} \int_{t}^{t+\Delta} B(u, T) d W^{r}(u)
\end{aligned}
$$

where $W_{j}(u)$ are Brownian motions independent of $W_{j}^{V}(u)$ and $W^{r}(u)$.

By the drift interpolation method [25], we approximate the integral of the variance process $V_{j}(t)(j=1,2)$ by

$$
\int_{t}^{t+\Delta} V_{j}(u) d u \approx \frac{1}{2}\left[V_{j}(t)+V_{j}(t+\Delta)\right] \Delta
$$

By (7) and (8), we have

$$
\begin{aligned}
& \int_{t}^{t+\Delta} \sqrt{V_{j}(u)} d W_{j}^{V}(u)=\frac{1}{\sigma_{j}}\left[V_{j}(t+\Delta)-V_{j}(t)\right. \\
& \left.-b_{j} \theta_{j} \Delta+b_{j} \int_{t}^{t+\Delta} V_{j}(u) d u\right] .
\end{aligned}
$$

The integral $\int_{t}^{t+\Delta} \sqrt{V_{j}(u)} d W_{j}(u)(j=1,2)$ obeyed normal distribution with mean zero and variance $\int_{t}^{t+\Delta} V_{j}(u) d u$. By (16), we approximate $\int_{t}^{t+\Delta} \sqrt{V_{j}(u)} d W_{j}(u)$ by

$$
\int_{t}^{t+\Delta} \sqrt{V_{j}(u)} d W_{j}(u) \approx \sqrt{\frac{1}{2}\left[V_{j}(t)+V_{j}(t+\Delta)\right] \Delta} Z_{j},
$$

where $Z_{j}$ are independent standard normal random variables.

By direct calculation, we have

$$
\begin{gathered}
\int_{t}^{t+\Delta} B^{2}(u, T) d u=\frac{1}{b_{r}^{2}}\left(\Delta+\frac{e^{-2 b_{r}(T-t-\Delta)}-e^{-2 b_{r}(T-t)}}{2 b_{r}}\right. \\
\left.+\frac{2 e^{-b_{r}(T-t)}-2 e^{-b_{r}(T-t-\Delta)}}{b_{r}}\right)=C(t, t+\Delta) .
\end{gathered}
$$

Accordingly, we have the integral $\int_{t}^{t+\Delta} B(u, T) d W^{r}(u)$ obeying normal distribution with mean zero and variance $\int_{t}^{t+\Delta} B^{2}(u, T) d u$; that is,

$$
\int_{t}^{t+\Delta} B(u, T) d W^{r}(u)=\sqrt{C(t, t+\Delta)} Z_{r}
$$

where $Z_{r}$ is a standard normal random variable.

Substituting (16)-(20) into (15), we have the simulation scheme for the asset price process as follows:

$$
\begin{aligned}
z(t+\Delta, T)= & z(t, T)+K_{0} \\
& +\sum_{j=1}^{2}\left[K_{1}^{j} V_{j}(t)+K_{2}^{j} V_{j}(t+\Delta)+K_{3}^{j} Z_{j}\right] \\
& +K_{4} Z_{r},
\end{aligned}
$$

where

$$
\begin{aligned}
& K_{0}=-\frac{\sigma_{r}^{2}}{2 b_{r}^{2}}\left(\Delta+\frac{e^{-2 b_{r}(T-t-\Delta)}}{2 b_{r}}-\frac{2 e^{-b_{r}(T-t-\Delta)}}{b_{r}}-\frac{e^{-2 b_{r}(T-t)}}{2 b_{r}}+\frac{2 e^{-b_{r}(T-t)}}{b_{r}}\right) \\
& -\frac{b_{j} \theta_{j} \rho_{j} \Delta}{\sigma_{j}}, \\
& K_{1}^{j}=-\frac{\Delta}{4}+\frac{b_{j} \rho_{j} \Delta}{2 \sigma_{j}}-\frac{\rho_{j}}{\sigma_{j}}, \\
& K_{2}^{j}=-\frac{\Delta}{4}+\frac{b_{j} \rho_{j} \Delta}{2 \sigma_{j}}+\frac{\rho_{j}}{\sigma_{j}},
\end{aligned}
$$


(1) Initial $K, S, B, T, r_{0}, b_{r}, \sigma_{r}, b_{j}, \theta_{j}, \sigma_{j}, \rho_{j}, V_{j}, \psi_{c}$;

(2) Compute $P(0, T)$ using (4);

(3) sum $_{-} p=0$;

(4) repeat for $i=1$ to $M$

(5) Compute $m_{j}, s_{j}^{2}, \psi_{j}, \alpha_{j}, \beta_{j}$ for $j=1,2$;

(6) Draw two uniform random numbers $U_{j}$;

(7) Draw two standard normal random numbers $Z_{V j}$ by $\Phi^{-1}\left(U_{j}\right)$;

(8) if $\psi_{j} \leq \psi_{c}$ then compute $V_{j}(t)$ by $\alpha_{j}\left(\beta_{j}+Z_{V j}\right)^{2}$;

(9) else compute $V_{j}(t)$ by (14);

(10) end if

(11) Compute $K_{0}, K_{1}^{j}, K_{2}^{j}, K_{3}^{j}, K_{4}$;

(12) Compute $z(t)$ by (21);

(13) $\quad$ Set $S(t)=P(t, T) e^{z(t)}$;

(14) $\quad$ if $S \leq B$, then payoff $=0$;

(15) else payoff $=\max (S-K, 0)$;

(16) end if

(17) sum_ $_{-} p=$ sum $_{-} p+$ payoff;

(18) until the last simulation

(19) Compute $V_{\text {DOC }}(0)$ by $V_{\text {DOC }}(0)=P(0, T)$ sum $p / M$.

Algorithm 1: The basic algorithm for pricing a DOC option.

$$
\begin{aligned}
& K_{3}^{j}=\sqrt{\frac{\Delta}{2}\left(1-\rho_{j}^{2}\right)\left[V_{j}(t)+V_{j}(t+\Delta)\right]}, \\
& K_{4}=\sqrt{\frac{\sigma_{r}^{2}}{b_{r}^{2}}\left(\Delta+\frac{e^{-2 b_{r}(T-t-\Delta)}}{2 b_{r}}-\frac{2 e^{-b_{r}(T-t-\Delta)}}{b_{r}}-\frac{e^{-2 b_{r}(T-t)}}{2 b_{r}}+\frac{2 e^{-b_{r}(T-t)}}{b_{r}}\right)} .
\end{aligned}
$$

\section{Simulation Algorithm for Pricing Barrier Options}

Under the $T$-forward measure $Q$, we evaluate barrier options by the following formula:

$$
V_{b}(t, T)=P(t, T) E^{Q}\left[\Lambda\left(S\left(\tau_{B}\right), \tau_{B}\right)\right],
$$

where $\Lambda\left(S\left(\tau_{B}\right), \tau_{B}\right)$ is a payoff function, $B$ is barrier level, and $\tau_{B}$ is the first time when barrier is hit. For a down and out call (DOC) option $\tau_{B}$ is defined as follows:

$$
\tau_{B}=\inf \{0 \leq t \leq T: S(t)>B\} .
$$

$\Lambda\left(S\left(\tau_{B}\right), \tau_{B}\right)$ is given by

$$
\Lambda\left(S\left(\tau_{B}\right), \tau_{B}\right)= \begin{cases}\max (S(T)-K, 0), & \tau_{B}=T \\ 0, & \tau_{B}<T,\end{cases}
$$

where $K$ is exercise price at maturity time $T$.

4.1. The Basic Algorithm. Based on the simulation scheme for the DHHW model, we evaluate a DOC option by Algorithm 1.
4.2. The Hybrid Algorithm Based on Variance Reduction Techniques. We combine antithetic variate and control variate techniques to improve the efficiency of the Algorithm 1. We use a European option price as control variate and modify step (19) of Algorithm 1 as follows:

$$
\begin{aligned}
\widehat{V}_{\text {DOC }}(0) & \\
= & \frac{P(0, T) s_{-1} p}{M} \\
& +\frac{1}{M} \sum_{j=1}^{M}\left[V_{E}(0)-P(0, T) \max (S(T)-K, 0)\right],
\end{aligned}
$$

where $V_{E}(0)$ is the exact price of the European call option which can be efficiently calculated by the fast Fourier transform method [26, 27]. Given the characteristic function, $V_{E}(0)$ can be approximated by the following formula:

$$
\begin{aligned}
V_{E}(0) & \approx \frac{\exp (-\alpha k)}{\pi} \sum_{j=1}^{N} e^{-i(2 \pi / N)(j-1)(i-1)} e^{i b v_{j}} \psi\left(v_{j}\right) \\
\cdot & \frac{\eta}{3}\left[3+(-1)^{j}-\delta_{j-1}\right],
\end{aligned}
$$


TABLE 1: Estimated parameter values for the DHHW model calibrated to S\&P 500 index call options on March 28, 2017.

\begin{tabular}{llclr}
\hline \multicolumn{5}{c}{ Parameter values } \\
\hline$b_{r}=0.201$ & $\sigma_{r}=0.021$ & $r_{0}=0.012$ & & \\
$b_{1}=0.101$ & $\theta_{1}=0.019$ & $\sigma_{1}=0.189$ & $V_{1}=0.011$ & $\rho_{1}=-0.251$ \\
$b_{2}=2.114$ & $\theta_{2}=0.012$ & $\sigma_{2}=0.253$ & $V_{2}=0.021$ & $\rho_{2}=-0.823$ \\
\hline
\end{tabular}

where $k=\log K, \alpha$ is damping factor, $i$ is an imaginary unit, $b=N h / 2, \eta=2 \pi / N h$ ( $h$ is a regular spacing), $\delta_{n}$ is the Kronecker delta function that is unity for $n=0$ and zero otherwise, and $\psi(v)=\varphi(v-(\alpha+1) i) /\left(\alpha^{2}+\alpha-v^{2}+i(2 \alpha+1) v\right)$, where $\varphi(u)=E^{P}\left[e^{-\int_{t}^{T} r(u) d u+i u \ln S(t)}\right]$ is the discounted $T$ forward characteristic function of $\log S(t)$.

Based on the results of Grzelak et al. [14] and simple calculation, we have the discounted $T$-forward characteristic function of the DHHW model as follows:

$$
\varphi(u)=e^{i u \ln S(t)+D(u, \tau)+\sum_{j=1}^{2} E_{j}(u, \tau) V_{j}(t)+F(u, \tau) r(t)},
$$

where

$$
\begin{aligned}
& D(u, \tau)=(i u-1)\left[-B(t, T) \gamma_{t}+\ln \frac{P(0, t)}{P(0, T)}\right. \\
& \left.+\frac{1}{2}(H(0, T)-H(0, t))\right]-\frac{1}{2}\left(u^{2}-1+2 i u\right) \\
& \cdot H(t, T)+\frac{b_{j} \theta_{j}}{\sigma_{j}^{2}}\left[\left(b_{j}-\rho_{j} \sigma_{j} i u-D_{j}\right) \tau\right. \\
& \left.-2 \ln \left(\frac{1-G_{j} e^{-D_{j} \tau}}{1-G_{j}}\right)\right] \text {, } \\
& E_{j}(u, \tau)=\frac{b_{j}-\rho_{j} \sigma_{j} i u-D_{j}}{\sigma_{j}^{2}}\left(\frac{1-e^{-D_{j} \tau}}{1-G_{j} e^{-D_{j} \tau}}\right), \\
& F(u, \tau)=\frac{1}{b_{r}}(i u-1)\left(1-e^{-b_{r} \tau}\right), \\
& H(t, T)=\frac{\sigma_{r}^{2}}{b_{r}^{2}}\left(T-t+\frac{2}{b_{r}} e^{-b_{r}(T-t)}-\frac{1}{2 b_{r}} e^{-2 b_{r}(T-t)}\right. \\
& \left.-\frac{3}{2 b_{r}}\right) \\
& \gamma_{t}=f(0, t)+\frac{\sigma_{r}^{2}\left(1-e^{-b_{r} t}\right)^{2}}{2 b_{r}^{2}}, \\
& G_{j}=\frac{b_{j}-\rho_{j} \sigma_{j} i u-D_{j}}{b_{j}-\rho_{j} \sigma_{j} i u+D_{j}}, \\
& D_{j}=\sqrt{\left(b_{j}-\rho_{j} \sigma_{j} i u\right)^{2}+\sigma_{j}^{2}\left(u^{2}+i u\right)}, \\
& \tau=T-t .
\end{aligned}
$$

For computing $\Phi^{-1}\left(U_{j}\right)$ in step (7) of Algorithm 1, we use the approximation algorithm of Wichura [28] not by the Matlab function norminv.m. This can greatly decrease the simulation time. With the obtained $Z_{V j}$, we consider antithetic variable $-Z_{V j}$ and compute $V_{j}(t)$ by $\alpha_{j}\left(\beta_{j}-Z_{V j}\right)^{2}$ and thus obtain the second path of the DHHW model. By combining control variate technique we modify the last step of Algorithm 1 as

$$
\begin{aligned}
\widehat{V}_{\text {DOC }}(0) & \\
= & \frac{P(0, T) 0.5\left(\text { sum }_{-} p 1+\text { sum }_{-} p 2\right)}{M} \\
& +\frac{1}{M} \sum_{j=1}^{M}\left[V_{E}(0)-P(0, T) \max (S(T)-K, 0)\right] .
\end{aligned}
$$

\section{Numerical Experiments}

5.1. Calibration of the Model. Before applying the DHHW model to option pricing, we need to estimate the model parameters. For this purpose we use financial market data to calibrate the DHHW model. We define the following relative mean squares error (RMSE):

$$
\mathrm{RMSE}=\frac{1}{N T \times N K} \sum_{t=1}^{N T} \sum_{k=1}^{N K} \frac{\left(V_{t k}^{\Theta}-V_{t k}\right)^{2}}{V_{t k}},
$$

where $V_{t k}^{\Theta}$ and $V_{t k}$ are the model and market prices, respectively. We calibrate the DHHW model by solving the following nonlinear least-squares optimization problem:

$$
\Theta^{*}=\arg \min \mathrm{RMSE} \text {. }
$$

We choose the S\&P 500 index call options on March 28, 2017, available online at http://www.cboe.com/. The options have maturities between 366 days and 997 days and strike prices ranging from 2225 to 2500 . The closing price of underlying is 2358.57 . For the sake of simplicity, we set the dividend yield to zero. We calculate the model prices by (26). We use differential evolution algorithm (global optimum) to seek the optimal parameter set $\Theta^{*}$. Table 1 lists the calibrated parameter values and associated RMSE.

5.2. Implementation of the Simulation Algorithm. With the obtained parameter values we first test the performance of the simulation scheme. We apply the simulation scheme to European options and take the fast Fourier transform solutions as benchmark. In (26) we use $N=4096, h=$ $\pi / 300=0.01047$, and $\alpha=1.25$ and set $S=100, K=100$, and $T=2$. For our simulation scheme, we specify $\psi_{c}=$ 1.5 throughout the paper. We use 1000, 10000, and 100000 
TABLE 2: Comparison of the accuracy of our simulation scheme and Euler scheme for pricing European options. The exact price $V=13.209$.

\begin{tabular}{lcccc}
\hline \multirow{2}{*}{\begin{tabular}{l} 
Number of simulations \\
\cline { 2 - 4 }
\end{tabular}} & Number of time steps & \multicolumn{2}{c}{ Our simulation scheme } & \multicolumn{2}{c}{ Euler scheme } \\
Relative error
\end{tabular}

TABLE 3: Comparison of the standard errors among the different Monte Carlo techniques for pricing DOC options, where time steps $N=100$ and barrier $B=90$

\begin{tabular}{cccccc}
\hline$K$ & $M$ & Basic algorithm & Control variates & Antithetic variates & Hybrid algorithm \\
\hline \multirow{2}{*}{80} & 1000 & 0.6548 & 0.3167 & 0.4248 & 0.1047 \\
& 10000 & 0.2079 & 0.1012 & 0.1334 & 0.0342 \\
100 & 100000 & 0.0657 & 0.0312 & 0.0428 & 0.0114 \\
& 1000 & 0.4671 & 0.2317 & 0.3323 & 0.0112 \\
& 10000 & 0.1502 & 0.0689 & 0.0355 & 0.0248 \\
120 & 100000 & 0.0478 & 0.0219 & 0.2454 & 0.0077 \\
& 1000 & 0.2843 & 0.1418 & 0.0850 & 0.0456 \\
& 10000 & 0.0929 & 0.0437 & 0.0276 & 0.0152 \\
\end{tabular}

simulation trials, respectively. For each simulation trial we use 100 and 500 time steps, respectively. Table 2 compares our simulation scheme with the standard Euler scheme through sample standard errors and relative errors.

Table 2 shows that although there is almost no difference in the sample standard error between the two schemes, significant relative error remains in Euler scheme compared to our simulation scheme. Moreover, it is observed that standard error of our simulation scheme decreases with the increase of the number of simulations and is almost unaffected by time steps. Table 2 implies that our simulation scheme outperforms the Euler scheme.

With the same parameter setting we evaluate the DOC options with barrier $B=90$ by the hybrid algorithm. We still use 1000, 10000, and 100000 simulation trials. Since our simulation scheme is almost unaffected by time steps we only use 100 time steps. Table 3 compares the hybrid algorithm with basic algorithm, control variates, and antithetic variates techniques. The criterion used is sample standard errors produced by the above methods. Table 3 shows that both control variates and antithetic variates can reduce standard errors. The hybrid algorithm which combines the two techniques significantly reduces the standard errors. Table 3 implies that the hybrid algorithm is efficient for pricing barrier options.

Furthermore, based on the hybrid algorithm, we plot the implied volatility surface of the DHHW model. Figure 1 examines the effects of interest rate parameters $b_{r}, \sigma_{r}$ and the second variance parameters $b_{2}, \theta_{2}, \sigma_{2}, \rho_{2}$ on the implied volatility surface. To examine the effects of the above parameters we change each parameter by setting three different values while fixing all other parameter values.

Figure 1 summarizes our findings. Both $b_{r}$ and $\sigma_{r}$ only affect the long end regarding the maturity of the volatility surface. However, decreasing $b_{r}$ leads to a significant rise of the slope of the implied volatility smile, while the contrary occurs by decreasing $\sigma_{r}$. Increasing $b_{2}$ results in a fall of volatility, and this effect on the short-term volatility is more significant, which leads to the slight decrease of the curvature of the smile. Increasing $\theta_{2}$ results in the rise of overall volatility, but the slope of the smile hardly changed. The curvature of the smile slightly increases by increasing $\sigma_{2}$. By changing $\rho_{2}$, the slope of the smile significantly changes and a negative slope is displayed in the implied volatility. Figure 1 implies that interest rates and the second variance process have important effects on the implied volatility surface of the DHHW model.

\section{Conclusion}

We propose the DHHW model by combining the double Heston stochastic volatility and Hull-White stochastic interest rate. Under the $T$-forward measure, this paper develops a simulation scheme for the DHHW model. Combining the control variates and antithetic variates, this paper provides a hybrid Monte Carlo algorithm for pricing barrier options under the DHHW model. Numerical results show that the 

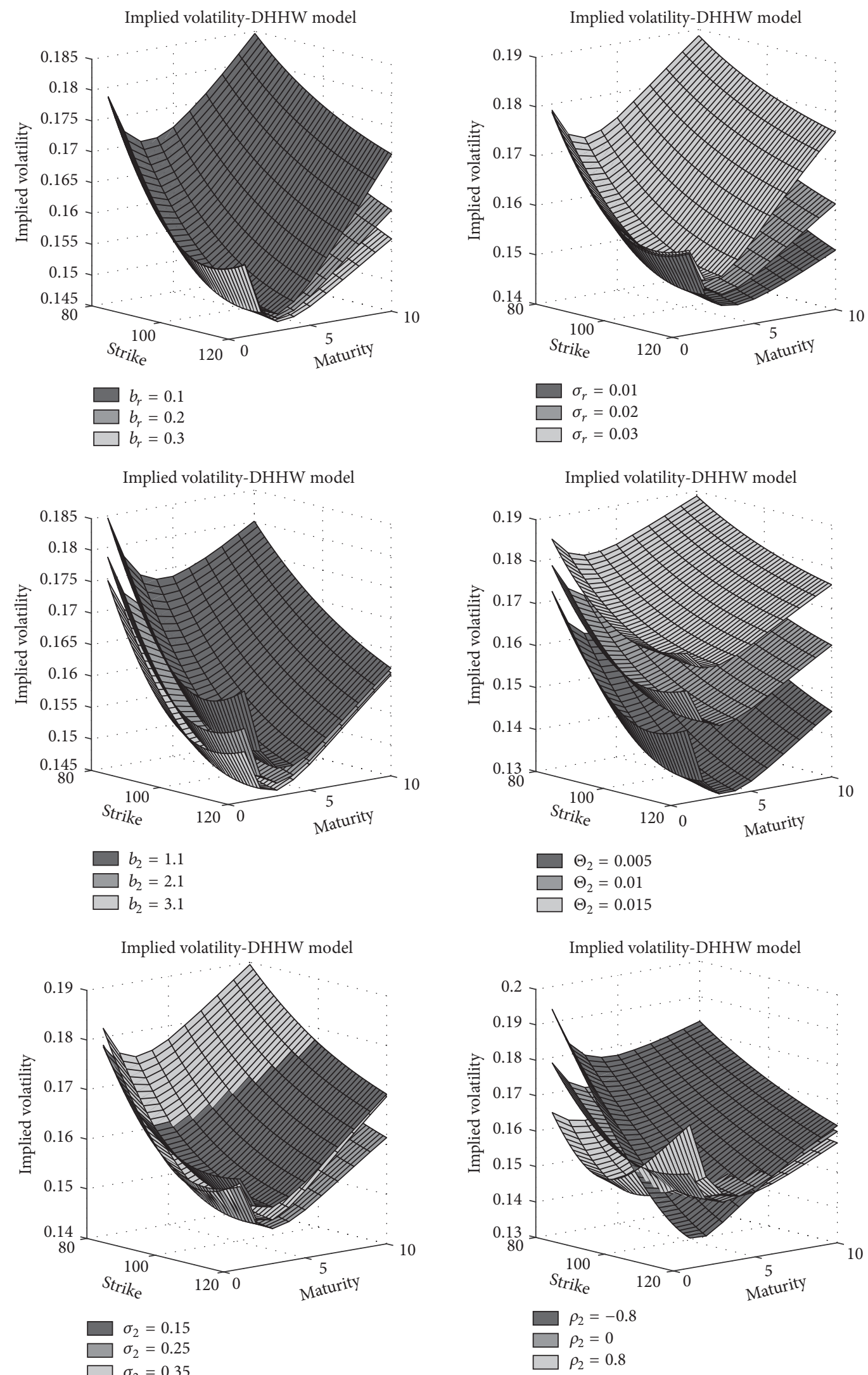

FIGURE 1: Implied volatility surface under the DHHW model with different values of parameters $b_{r}, \sigma_{r}, b_{2}, \theta_{2}, \sigma_{2}$, and $\rho_{2}$. 
proposed scheme outperforms Euler scheme and the hybrid algorithm is efficient and easy to implement in pricing barrier options. Extensive implied volatility experiments show that implied volatility surfaces present different shapes by varying the parameter values of interest rate and the second variance process, which verify that the DHHW model is flexible to fit the implied volatility smile.

\section{Conflicts of Interest}

The authors declare that there are no conflicts of interest regarding the publication of this paper.

\section{Acknowledgments}

The authors would like to acknowledge the support from the National Natural Science Foundation of China (Grant no. 11601420), the Natural Science Basic Research Plan in Shaanxi Province of China (Grant no. 2017JM1021), and the Scientific Research Program Funded by Shaanxi Provincial Education Department (Grant no. 17JK0714).

\section{References}

[1] R. Cont and P. Tankov, Financial Modelling with Jump Processes, Chapman \& Hall, CRC Press, London, UK, 2004.

[2] P. Christoffersen, S. Heston, and K. Jacobs, "The shape and term structure of the index option smirk: why multifactor stochastic volatility models work so well," Management Science, vol. 55, no. 12, pp. 1914-1932, 2009.

[3] M. Yousuf, "A fourth-order smoothing scheme for pricing barrier options under stochastic volatility," International Journal of Computer Mathematics, vol. 86, no. 6, pp. 1054-1067, 2009.

[4] M. Yousuf, "Efficient smoothing of Crank-Nicolson method for pricing barrier options under stochastic volatility," PAMM, vol. 7, no. 1, pp. 1081101-1081102, 2007.

[5] S. A. Griebsch and U. Wystup, "On the valuation of fader and discrete barrier options in Heston's stochastic volatility model," Quantitative Finance, vol. 11, no. 5, pp. 693-709, 2011.

[6] Y. Tian, Z. Zhu, F. C. Klebaner, and K. Hamza, "Pricing barrier and American options under the SABR model on the graphics processing unit," Concurrency and Computation: Practice and Experience, vol. 24, no. 8, pp. 867-879, 2012.

[7] K. Shiraya, A. Takahashi, and T. Yamada, "Pricing discrete barrier options under stochastic volatility," Asia-Pacific Financial Markets, vol. 19, no. 3, pp. 205-232, 2012.

[8] J. Jeon, J.-H. Yoon, and C.-R. Park, "An analytic expansion method for the valuation of double-barrier options under a stochastic volatility model," Journal of Mathematical Analysis and Applications, vol. 449, no. 1, pp. 207-227, 2017.

[9] H. Funahashi and T. Higuchi, "An analytical approximation for single barrier options under stochastic volatility models," Annals of Operations Research, vol. 6, pp. 1-29, 2017.

[10] S. L. Heston, "A closed-form solution for options with stochastic volatility with applications to bond and currency options," The Review of Financial Studies, vol. 6, no. 2, pp. 327-343, 1993.

[11] J. Gatheral, The Volatility Surface: A Practitioner's Guide, John Wiley \& Sons, Inc., Hoboken, NJ, USA, 2012.

[12] P. Gauthier and D. Possamai, "Efficient simulation of the double heston model," IUP Journal of Computational Mathematics, vol. 4, no. 3, pp. 23-75, 2011.
[13] A. Göncü and G. Ökten, "Efficient simulation of a multifactor stochastic volatility model," Journal of Computational and Applied Mathematics, vol. 259, no. 6, pp. 329-335, 2014.

[14] L. A. Grzelak, C. W. Oosterlee, and S. Van Weeren, "Extension of stochastic volatility equity models with the Hull-White interest rate process," Quantitative Finance, vol. 12, no. 1, pp. 89-105, 2012.

[15] B. Chen, L. A. Grzelak, and C. W. Oosterlee, "Calibration and Monte Carlo pricing of the SABR-Hull-White model for long-maturity equity derivatives," The Journal of Computational Finance, vol. 15, no. 4, pp. 79-113, 2012.

[16] S. Guo, L. A. Grzelak, and C. W. Oosterlee, "Analysis of an affine version of the Heston-Hull-White option pricing partial differential equation," Applied Numerical Mathematics, vol. 72, no. 1, pp. 143-159, 2013.

[17] S. Simaitis, C. S. de Graaf, N. Hari, and D. Kandhai, "Smile and default: the role of stochastic volatility and interest rates in counterparty credit risk," Quantitative Finance, vol. 16, no. 11, pp. 1725-1740, 2016.

[18] C. Chiarella, B. Kang, and G. H. Meyer, "The evaluation of barrier option prices under stochastic volatility," Computers \& Mathematics with Applications, vol. 64, no. 6, pp. 2034-2048, 2012.

[19] Y. L. Hsiao, S. Y. Shen, and A. M. Wang, "A hybrid finite difference method for pricing two-asset double barrier options," Mathematical Problems in Engineering, vol. 2015, Article ID 692695, 7 pages, 2015.

[20] P. Shevchenko and P. Del Moral, "Valuation of barrier options using sequential Monte Carlo," The Journal of Computational Finance, vol. 20, no. 4, pp. 107-135, 2016.

[21] F. Cong and C. W. Oosterlee, "Multi-period mean-variance portfolio optimization based on Monte-Carlo simulation," Journal of Economic Dynamics \& Control, vol. 64, pp. 23-38, 2016.

[22] J. Hull and A. White, "Pricing interest-rate derivative securities," Review of Financial Studies, vol. 3, no. 4, pp. 573-592, 1990.

[23] D. Brigo and F. Mercurio, Interest Rate Models: Theory and Practice: With Smile, Inflation and Credit, Springer Finance, Springer, New York, NY, USA, 2nd edition, 2006.

[24] L. Andersen, "Efficient simulation of the Heston stochastic volatility model," Journal of Computational Finance, vol. 11, no. 3, pp. 1-42, 2008.

[25] D. Dufresne, The Integrated Square-Root Process, Working paper, University of Montreal, 2001.

[26] P. Carr, M. Stanley, and D. B. Madan, "Option valuation using the fast Fourier transform," The Journal of Computational Finance, vol. 2, no. 4, pp. 61-73, 1999.

[27] S.-M. Zhang and L.-H. Wang, "A fast Fourier transform technique for pricing European options with stochastic volatility and jump risk," Mathematical Problems in Engineering, vol. 2012, Article ID 761637, 2012.

[28] M. J. Wichura, "Algorithm AS 241: the percentage points of the normal distribution," Journal of Applied Statistics, vol. 37, no. 3, pp. 477-484, 1988. 


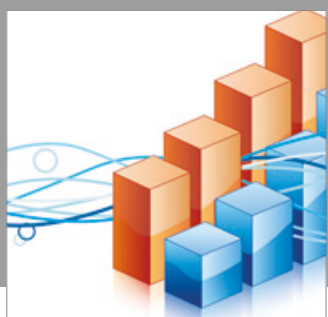

Advances in

Operations Research

vatersals

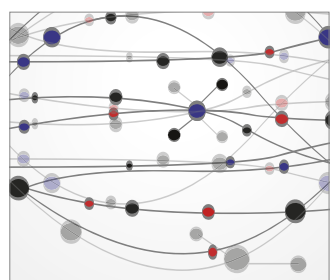

\section{The Scientific} World Journal
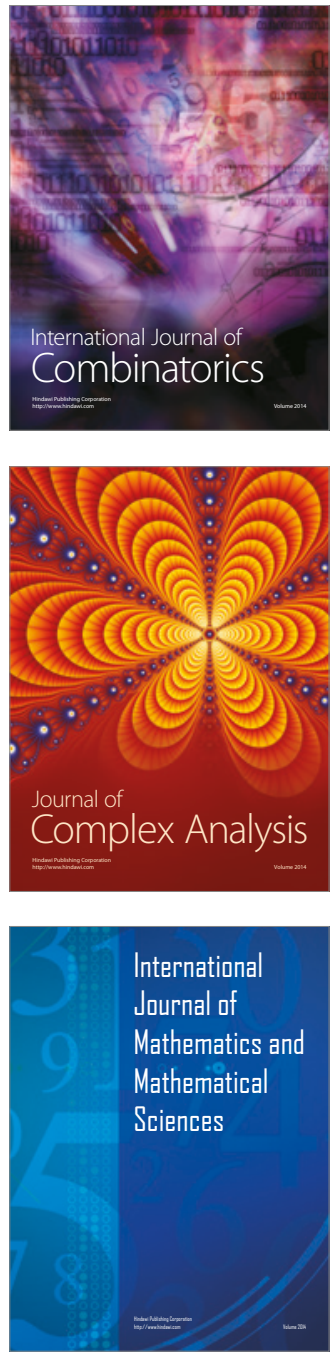
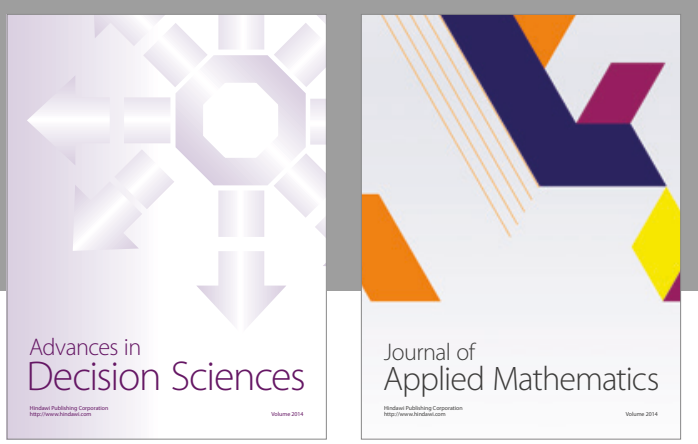

Algebra

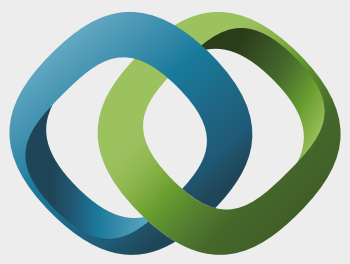

\section{Hindawi}

Submit your manuscripts at

https://www.hindawi.com
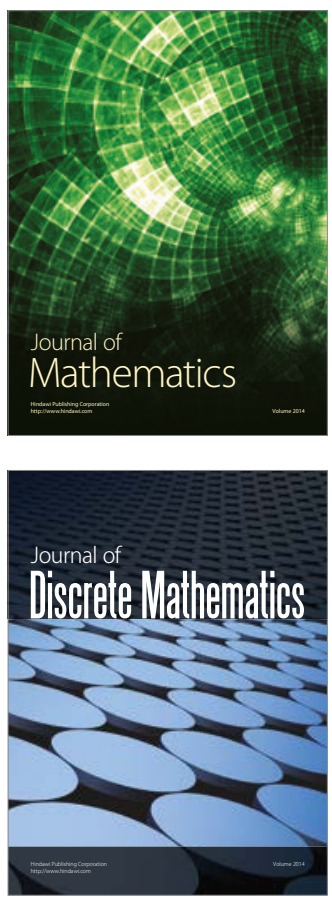

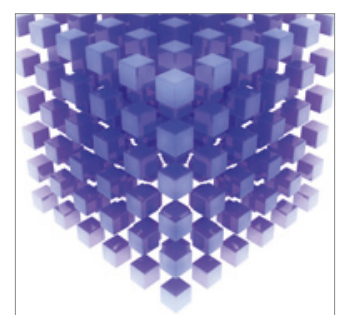

Mathematical Problems in Engineering
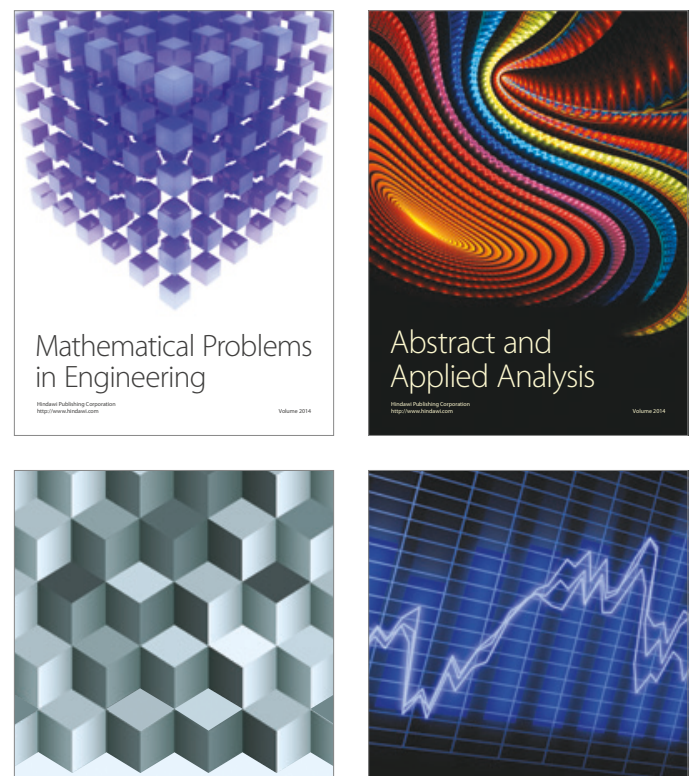

Journal of

Function Spaces

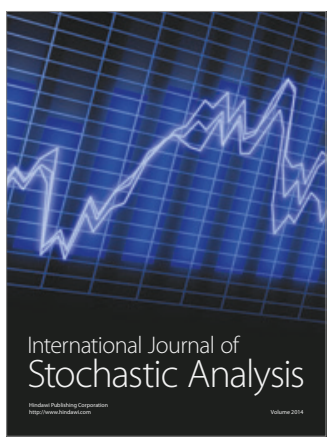

Probability and Statistics
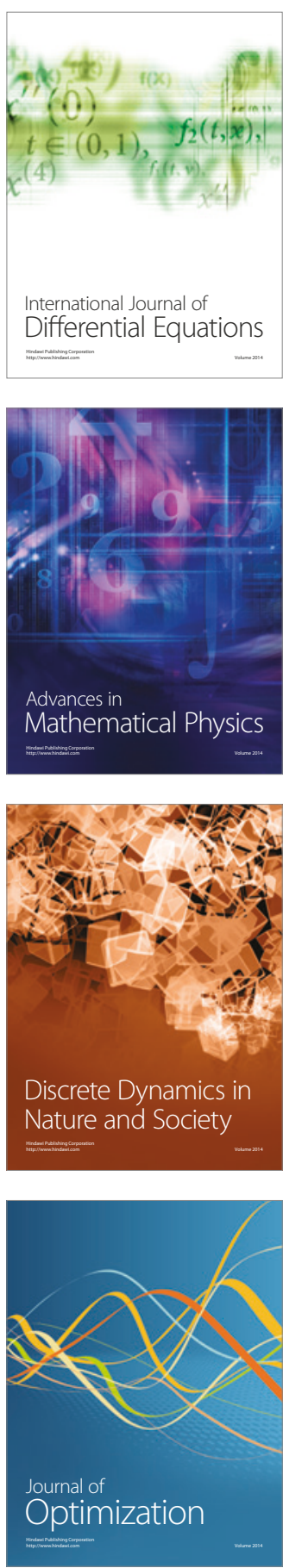